Table 1 Results of GeneXpert Xpress SARS-CoV-2 assay pooling of clinical specimens

\begin{tabular}{lcc}
\hline Pooling protocol & $\begin{array}{c}\text { Average } \Delta \text { pool Ct } \\
\text { E gene }\end{array}$ & $\begin{array}{c}\text { Average } \Delta \text { pool Ct } \\
\text { N2 gene }\end{array}$ \\
\hline 4 to 1 & 1.9 & 2.7 \\
6 to 1 & 2.6 & 2.7 \\
8 to 1 & 3.3 & 3.5 \\
\hline
\end{tabular}

of four samples, where only one sample may be positive and, in effect, diluting the positive specimen 1:4. This result is consistent with the results of similar experiments described by Chong et al. $^{8}$ with an increase in $\mathrm{Ct}$ values following sample pooling, consistent with our own study despite the authors using a different SARS-CoV-2 assay. These authors also concluded that pools of four samples are the optimal pool size, trading assay sensitivity for a notable increase in the efficiency of sample testing. The latter study further concluded that this pooling strategy would be ideal for testing large numbers of samples with very low test-probability, even when there were no concerns over reagent access. ${ }^{8}$ Based on these results, we successfully introduced this sample pooling strategy as a component of our local diagnostic microbiology surge response to outbreaks of SARS-CoV-2 in Western Sydney between June and October 2021, reserving pooled specimens for lower risk patients. In the USA, specimen pooling has been approved as a strategy to increase screening testing capacity for SARS-CoV-2 assays approved for use by the FDA. ${ }^{0}$ Pooling of specimens on the GeneXpert platform is not currently endorsed by the manufacturer and laboratories are required to accredit this variation with NATA as inhouse in vitro diagnostic device (IVD). Of note, NSWHP laboratories are NATA accredited for pooling on several other SARS-CoV-2 NAT assays. The work described in this study, involving pragmatic changes to diagnostic procedures to conserve critical diagnostic reagents, highlights the importance of flexibility in managing a laboratory response to the challenges presented by COVID-19 and other new and emerging infectious disease threats now and into the future.

Conflicts of interest and sources of funding: The authors state that there are no conflicts of interest to disclose.

Rifky Balgahom ${ }^{1}$, Vishal Ahuja ${ }^{2}$, Catherine Pitman ${ }^{2}$, Adam Polkinghorne ${ }^{1,3}$, James Branley ${ }^{1,3}$

${ }^{1}$ Department of Microbiology and Infectious Diseases, New South Wales Health Pathology-Nepean, Penrith, NSW, Australia; ${ }^{2}$ New South Wales Health Pathology - Public Health Pathology State-wide Service, Westmead, NSW, Australia; ${ }^{3}$ Nepean Clinical School, Faculty of Medicine and Health, University of Sydney, Kingswood, NSW, Australia

Contact Dr James Branley.

E-mail: james.branley@health.nsw.gov.au

1. Jin Y, Yang H, Ji W, et al. Virology, epidemiology, pathogenesis, and control of COVID-19. Viruses 2020; 12: 372

2. D'Cruz RJ, Currier AW, Sampson VB. Laboratory testing methods for novel severe acute respiratory syndrome-coronavirus-2 (SARS-CoV-2). Front Cell Dev Biol 2020; 8: 468.
3. Loeffelholz MJ, Alland D, Butler-Wu SM, et al. Multicenter evaluation of the Cepheid Xpert xpress SARS-CoV-2 test. J Clin Microbiol 2020; 58: e00926-20.

4. COVID-19 National Incident Room Surveillance Team. COVID-19 Australia: epidemiology report 27. Fortnightly reporting period ending 11 October 2020. Commun Dis Intelligence 2020; 44: 1-20.

5. Mutesa L, Ndishimye P, Butera Y, et al. A pooled testing strategy for identifying SARS-CoV-2 at low prevalence. Nature 2020; 589: 27680 .

6. Volpato F, Lima-Morales D, Wink PL, et al. Pooling of samples to optimize SARS-CoV-2 diagnosis by RT-qPCR: comparative analysis of two protocols. Eur J Clin Microbiol Infect Dis 2020; 40: 889-92.

7. Cabrera Alvargonzalez JJ, Rey Cao S, Perez Castro S, et al. Pooling for SARS-CoV-2 control in care institutions. BMC Infect Dis 2020; 20: 745.

8. Chong BSW, Tran T, Druce J, Ballard SA, Simpson JA, Catton M. Sample pooling is a viable strategy for SARS-CoV-2 detection in lowprevalence settings. Pathology 2020; 52: 796-800.

9. Sparks R, Balgahom R, Janto C, Polkinghorne A, Branley J, Samarasekara H. An Australian diagnostic microbiology surge response to the COVID-19 pandemic. Diagn Microbiol Infect Dis 2021; 100: 115309

10. Centers for Disease Control and Prevention. Interim Guidance for Use of Pooling Procedures in SARS-CoV-2 Diagnostic, Screening, and Surveillance. Centers for Disease Control and Prevention, 2020.

DOI: https://doi.org/10.1016/j.pathol.2021.12.286

\section{Prolonged granulocyte transfusions sourced from buffy coats used to treat Aspergillus spp. infection in chronic granulomatous disease}

Sir,

Chronic granulomatous disease (CGD) is caused by inherited defects of the phagolysosomal NADPH-oxidase, leading to impaired neutrophil function and a high lifetime risk of invasive mould infection. We describe the successful outcome of an adult CGD patient with disseminated Aspergillus fischeri, with suspected triazole-resistance and clinical and radiological progression despite multiple high dose antifungals. His invasive fungal disease (IFD) was subsequently controlled by administering a prolonged course of granulocyte transfusions (GT) sourced from buffy coats (BCs).

A 37-year-old male with CGD (autosomal recessive $\mathrm{p} 47^{\text {phox }}$ deficiency) underwent donor-after-brain-death renal transplantation. Pre-transplantation, he had no active infections (including no evidence of latent mould infection on chest X-ray), and was receiving prophylactic itraconazole $200 \mathrm{mg}$ daily (Sporanox) and trimethoprim-sulfamethoxazole $160 / 800 \mathrm{mg}$ daily with therapeutic drug monitoring of itraconazole achieving serum levels between 100 and $300 \mu \mathrm{g} / \mathrm{L}$. The donor had no risk factors for mould infection. Immune suppression consisted of intravenous (IV) basiliximab and methylprednisolone perioperatively, followed by maintenance tacrolimus, mycophenolate mofetil and prednisolone as per standard institutional protocols.

His transplant was complicated by biopsy-confirmed graft rejection at 2 weeks, requiring pulse high-dose methylprednisolone followed by a prolonged weaning dose of oral prednisone. At 12 weeks he developed headaches, vomiting and visual disturbance. Brain magnetic resonance imaging revealed ring enhancing lesions in the left parietal and right occipital lobes suspicious for IFD (Fig. 1), requiring a 

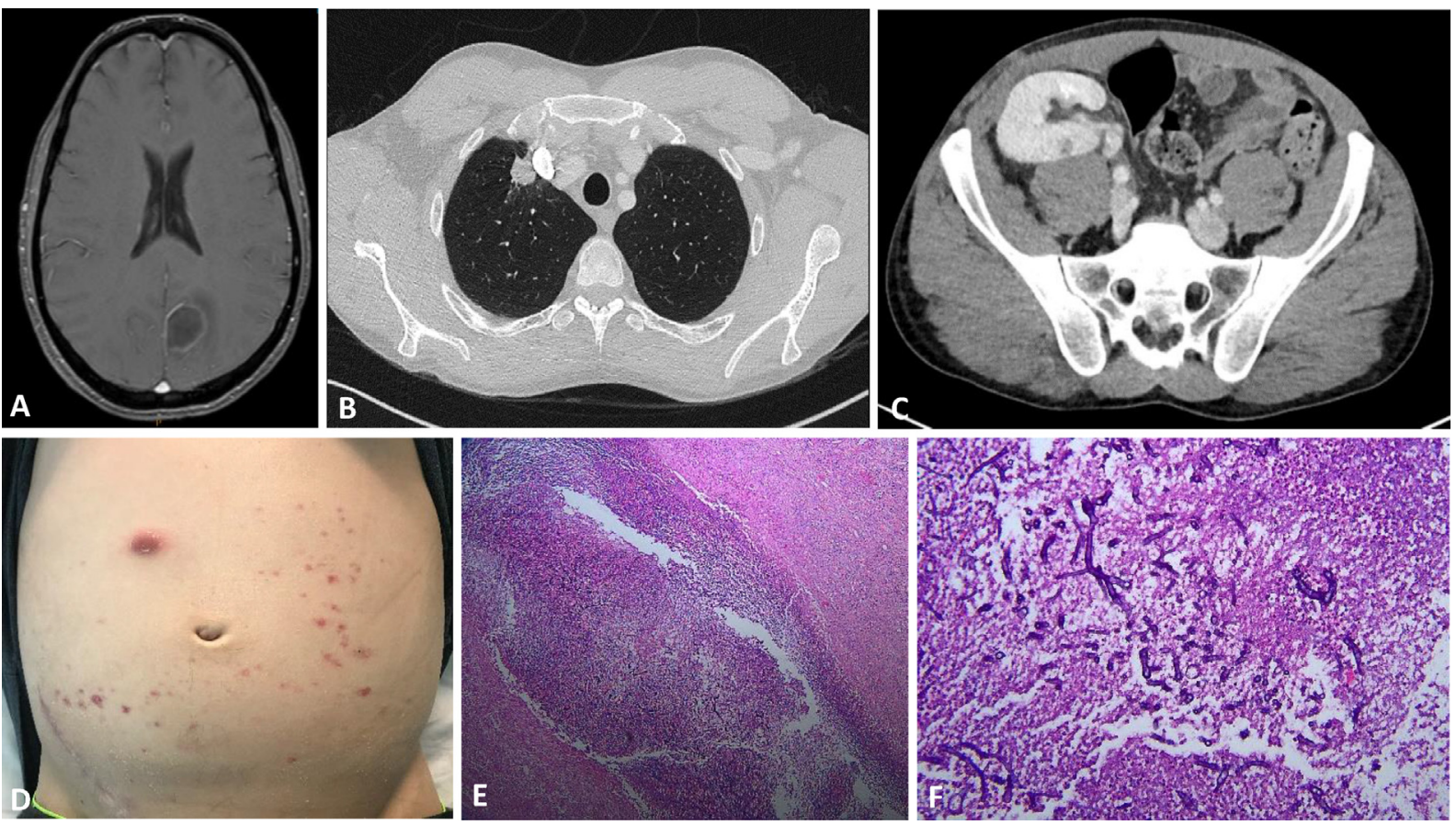

Fig. 1 (A) Left parietal lobe abscess. (B) Right upper lobe pulmonary nodular lesion. (C) Abscess in the right posterior cortex of transplanted kidney. (D) Multiple abdominal cutaneous fungal lesions. (E) H\&E stain of cutaneous fungal abscess. (F) H\&E stain of branching fungal hyphae in skin.

craniotomy for abscess evacuation and empirical commencement of IV liposomal amphotericin B $5 \mathrm{mg} / \mathrm{kg}$ Aspergillus fumigatus complex was cultured from the intraoperative samples taken from the left parietal lobe abscess and minimum inhibitory concentrations (MIC) were determined by broth microdilution (BMD) (Table 1). Clinical and Laboratory Standards Institute (CLSI) BMD standard for mould antifungal susceptibility testing was used.

Computed tomography (CT) chest, abdomen and pelvis revealed multiple lobulated lesions in both lungs (including cavitating nodules) and a small abscess within the posterior cortex of the transplant kidney. As this demonstrated high burden disseminated IFD, his antifungal therapy was upgraded to include voriconazole $200 \mathrm{mg}$ twice daily and caspofungin $140 \mathrm{mg}$ daily. Trough serum voriconazole levels ranged between 1.2 and $6.7 \mathrm{mg} / \mathrm{L}$, targeting between 4 and $5 \mathrm{mg} / \mathrm{L}$. Whilst on triple antifungal therapy he developed numerous raised tender erythematous nodular skin lesions over his trunk and limbs, many of which were biopsied with fungal cultures growing $A$. fumigatus complex. In the context of progressive IFD despite appropriate therapy, the patient's transplanted kidney was explanted to allow rapid

Table 1 Intra-operative Aspergillus fischeri isolates minimum inhibitory concentrations (MIC) in $\mathrm{mg} / \mathrm{L}$ determined by broth microdilution (BMD)

\begin{tabular}{lllllllllll}
\hline \multirow{2}{*}{ Isolate } & \multicolumn{8}{c}{ Antifungals tested } \\
\cline { 2 - 10 } & 5-FC & AMB & ANI & FLU & ITR & MIC & POS & VOR & ISA $^{\text {a }}$ \\
\hline Baseline & 64 & 2 & 0.015 & $>256$ & 1 & 0.015 & 1 & 2 & - \\
4 weeks & $>64$ & 2 & 0.015 & $>256$ & 0.5 & 0.015 & 0.5 & 2 & 0.75 \\
\hline
\end{tabular}

5-FC, Flucytosine; AMB, amphotericin; ANI, anidulafungin; FLU, fluconazole; ITR, itraconazole; MIC, micafungin; POS, posaconazole; VOR, voriconazole; ISA, isavuconazole.

${ }^{\text {a }}$ Performed by E-test method. wean of immune suppression; at the time, this consisted of tacrolimus $5 \mathrm{mg}$ twice daily, mycophenolate mofetil $750 \mathrm{mg}$ twice daily, and prednisolone $10 \mathrm{mg}$ daily. Post-operatively, tacrolimus and mycophenolate mofetil were ceased immediately, however he remained on dexamethasone $2 \mathrm{mg}$ daily to relieve abscess-induced intracranial hypertension. Despite these interventions, by 6 weeks post-presentation his IFD had progressed at all sites, evidenced by new abscesses in skin and lungs, and persisting brain abscesses requiring repeated washouts to relieve intracranial pressure; serial intra-cranial samples obtained up to 4 weeks after the index specimen continued to culture Aspergillus fumigatus complex with little change in MIC values (Table 1). Both intracranial A. fumigatus complex isolates taken at the first operation and at 4 weeks were sent for whole genome sequencing (WGS), which identified the species Aspergillus fischeri. Common cyp51A, cyp51B and cyp51C gene mutations (G54, L98, M220, P216, G138, and G448) conferring azole resistance were not identified in either isolate. Fungal genomes were sequenced on a NextSeq 500/550 platform with a NextSeq MID output $(2 \times 150$ bp $)$ sequencing kit (Illumina, USA). The genomes of Aspergillus fischeri were assembled using shovill (v1.0.9) ${ }^{1}$ and annotated using AUGUSTUS (v3.3.3). ${ }^{2}$ Species identification was performed by searching beta-tubulin gene (benA) from the draft genome against BLASTP database.

Given the lack of clinical response to cessation of immunosuppression and three broad-spectrum antifungals, the haematology service was consulted to assess the feasibility of serial GT as a potential adjunct therapy. Following combination granulocyte-colony stimulating factor (GCSF) and dexamethasone mobilisation, granulocytes were harvested by leukapheresis from three ABO-compatible family members, resulting in two blood volumes $(394-586 \mathrm{~mL})$ from each donation. Apheresis GT were collected and delivered as per institutional protocols. Each donor donated twice, and thus 
GT were administered to the patient twice weekly for 3 weeks while the patient continued to receive triple antifungal therapy. IFD restaging at this timepoint demonstrated substantial reduction in number and size of cutaneous lesions, stable pulmonary and intracerebral lesions, and cessation of fevers. Given this encouraging clinical response, the lack of immediate access to novel antifungal agents, and inability to collect from the same GT donor more than twice, the Australian Red Cross Lifeblood was engaged to provide ongoing GT support. In Australia, the BC granulocytes are not on the National Blood Product Supply list and are supplied under an individual patient tailored component request process.

ABO-identical [A Rh(D) positive, CMV irrelevant] BC GT were manufactured from routine whole blood donations on the same day as collection, irradiated, and infused on the same day as manufacture. Each single BC GT unit had a neutrophil count of $0.3-1.6 \times 10^{9} / \mathrm{L}$ within a volume of $46-$ $66 \mathrm{~mL}$. Initial dosing was intended as 10 units BC GT twice weekly, depending on product availability. During this time, triple antifungal therapy was progressively weaned and then ceased due to lack of efficacy and concerns regarding potential toxicity, and the patient transitioned to posaconazole $300 \mathrm{mg}$ monotherapy. Trough serum posaconazole levels ranged between 0.5 and $2.1 \mathrm{mg} / \mathrm{L}$, targeting $2 \mathrm{mg} / \mathrm{L}$. After 6 weeks of BC GT (62 units, median 7 per episode, range 310 ), all cutaneous lesions were eradicated, and all cardiopulmonary and intracerebral lesions had reduced by $>50 \%$ in size, which we interpreted as a partial response. Given this response, and due to the development of symptomatic polycythaemia from inadvertent passive transfer of red blood cells during BC GT, subsequent BC GT dosing was modified to accept 5-7 units twice weekly. Over the subsequent 14 weeks, the patient received 156 BC GT (median 6 per episode, range 2-10), ultimately ceasing to enrol in an antifungal clinical trial. By this point, the patient had achieved and maintained a stable partial response from IFD, after receiving six directed GT and 221 BC GT over 5 months. Figure 2 shows the timeline of events and patient response to treatment.

GT were well tolerated, with no episodes of febrile transfusion reaction or transfusion related acute lung injury (TRALI). At completion of the GT course, testing for anti- human neutrophil antigen antibodies was negative. No clinically significant donor-specific HLA antibodies were detected. Reattempt at renal transplantation with combined haematopoietic stem cell transplantation (HSCT) may be considered if IFD is eradicated.

Patients with CGD are susceptible to infections caused by catalase-positive microorganisms including Staphylococcus aureus, Nocardia spp. and Aspergillus spp., and are recommended to receive lifelong antimicrobial prophylaxis containing trimethoprim-sulfamethoxazole $(5 \mathrm{mg} / \mathrm{kg} / \mathrm{d}$ divided twice daily up to $320 \mathrm{mg}$ trimethoprim daily) and itraconazole $\left(5 \mathrm{mg} / \mathrm{kg} / \mathrm{d}\right.$ up to $200 \mathrm{mg}$ daily). ${ }^{3}$ Patients with CGD have a high lifetime incidence of invasive aspergillosis, and numerous cases of disseminated infection (predominantly involving CNS, pulmonary and bone) due to Aspergillus fumigatus or Aspergillus nidulans have been described, typically in the paediatric CGD population. Although itraconazole demonstrates good activity against Aspergillus spp., higher MICs have been observed among some species; this may warrant targeting high serum drug levels in the prophylaxis setting. In addition, itraconazole demonstrates poor penetration at some sites (e.g., central nervous system, eye) which may confer suboptimal protection. Although no common azole resistance genes were detected from our patient's isolates, azole MICs were above the epidemiological cut-off values (ECOFF) as outlined in the updated EUCAST clinical breakpoints against Aspergillus spp. (v10.0, 2020) where wild-type $A$. fumigatus posaconazole and voriconazole values were $0.25 \mathrm{mg} / \mathrm{L}$ and $1 \mathrm{mg} / \mathrm{L}$, respectively. ${ }^{4}$ This may partially explain failure of prophylaxis and a poor response to treatment with azole therapy.

Unusual clinical features of Aspergillus spp. infection have also been described in this unique population, including forearm myofasciitis and pericarditis. ${ }^{5,6}$ Mortality from disseminated aspergillosis in CGD is high; even more so when the CNS is involved. Survival has been associated with correction of the underlying immunodeficiency via adjunctive therapies including GT, interferon- $\gamma$, gene therapy and allogeneic HSCT.

GT was commonly used in the 1980s and 1990s to treat refractory infections in patients with chemotherapy-induced neutropenia or primary immunodeficiency, but their use has
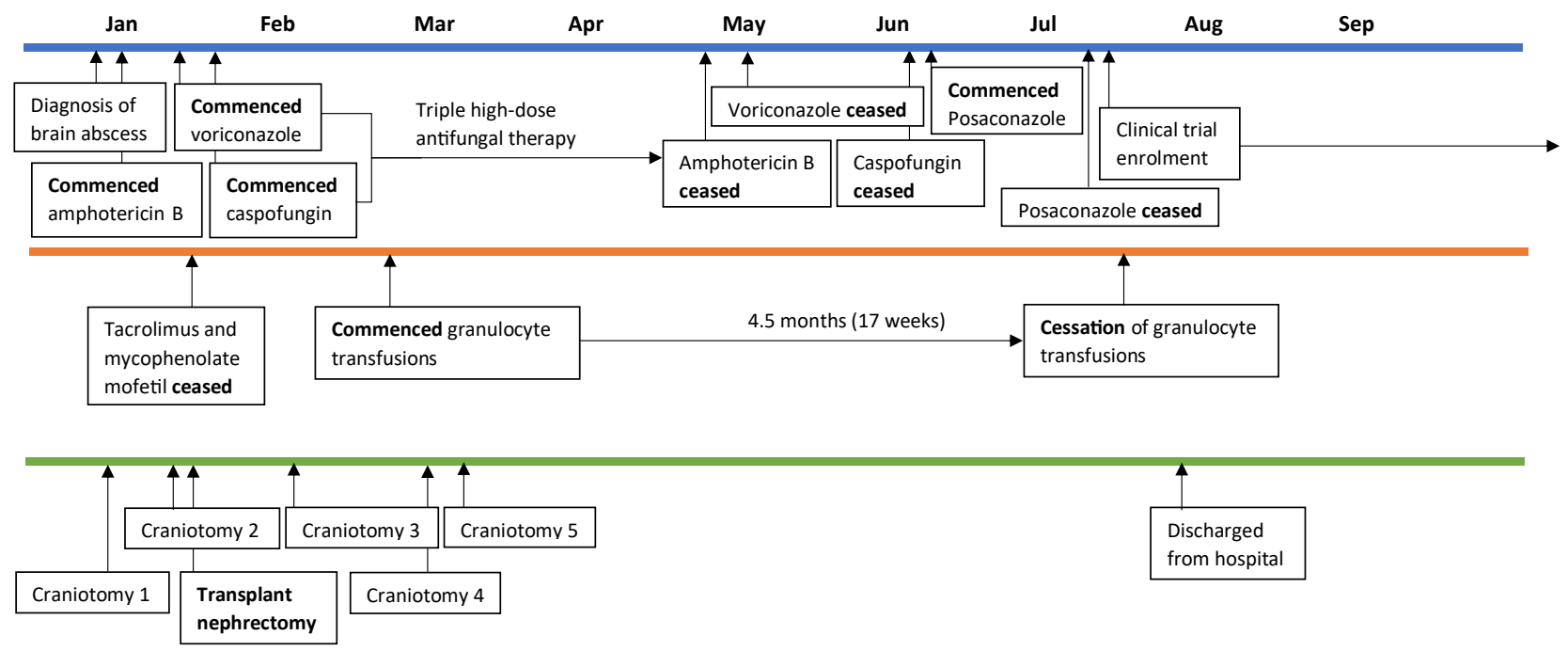

Fig. 2 Timeline of events and patient response to treatment. 
been substantially curtailed this millennium following the introduction of recombinant GCSF. ${ }^{7}$ Although no randomised study has successfully demonstrated a survival advantage for patients receiving GT, clinical outcomes have been reported in retrospective and prospective case series, suggesting that clinical improvement for bacterial infections can be achieved when sufficient GT neutrophil doses are delivered. However, outcomes for GT in IFD are heterogeneous at best, and have been reported as showing minimal clinical benefit and occasional potential detriment. ${ }^{8}$ There remains no consensus about the overall clinical efficacy of GT in the treatment of infection, perhaps representing a lack of evidence rather than lack of benefit.

While GT is occasionally used in crisis situations such as our patient's case, its clinical utility has often been limited by transfusion-related factors. Firstly, GT carries risks of immune-mediated transfusion reactions such as TRALI and transfusion-associated graft versus host disease (TA-GVHD). Secondly, the short lifespan of the transfused granulocytes, exacerbated by the irradiation performed to prevent TAGVHD, results in a need for repeated infusions to achieve clinical impact. Thirdly, GT source also impacts upon deliverability: although granulocyte yield is significantly higher from a directed-donation leukapheresis than from a single whole blood derived BC collection, extended GT treatment such as that required by our patient is rarely sustainable or feasible from directed donation, and is extremely labour-intensive for apheresis and transfusion services.

Reports of GT for IFD in CGD typically are sporadic doses given over a 1-4 week period, ceased either due to neutrophil recovery from prior chemotherapy, severe transfusion reaction, or patient death from IFD. The largest series in the literature reported a median 2-6 GT in 28 patients, resulting in an overall response rate of $82 \%$ with $1 \%$ incidence of transfusion reactions; ${ }^{9}$ the previously maximum reported dose and duration by any patient in the literature is three times a week for 2 months. ${ }^{10}$ Adoptive cell therapy (ACT), which involves the infusion of donor-derived pathogen-specific $\mathrm{T}$ cells, has been explored as a potential treatment option in patients with severe infections who are profoundly immunocompromised. ${ }^{11}$ This may be a future treatment modality for CGD patients with life-threatening IFD. Our case, which clearly exceeds the previous maximum reported treatment of GT for any infection, highlights the potential benefit for extended GT treatment in IFD, and the potential benefits in terms of utility and feasibility of using BC as GT source, without apparent compromise in terms of transfusion safety or alloimmunisation. However, this should only be limited to last resort therapy.

Acknowledgement: The Australian government funds the Australian Red Cross Lifeblood for the provision of blood, blood products and services to the Australian community.

Conflicts of interest and sources of funding: The authors state that there are no conflicts of interest to disclose.

\section{Adam G. Stewart ${ }^{1,2}$, Shoma Baidya ${ }^{3}$, James Daly ${ }^{3}$, Ashleigh P. Scott ${ }^{4}$}

${ }^{1}$ Department of Infectious Diseases, Royal Brisbane and Women's Hospital, Brisbane, Qld, Australia; ${ }^{2}$ Centre for Clinical Research, Faculty of Medicine, The University of
Queensland, Royal Brisbane and Women's Hospital Campus, Brisbane, Qld, Australia; ${ }^{3}$ Australian Red Cross Lifeblood, Brisbane, Qld, Australia; ${ }^{4}$ Department of Haematology and Bone Marrow Transplantation, Royal Brisbane and Women's Hospital, Brisbane, Qld, Australia

Contact Dr Adam G. Stewart.

E-mail: adam.stewart@uq.edu.au

1. Seeman T. Shovill. 2019; cited Feb 2021. https://github.com/tseemann/ shovill

2. Hoff KJ, Stanke M. Predicting genes in single genomes with AUGUSTUS. Curr Protoc Bioinform 2019; 65: e57.

3. Arnold DE, Heimall JR. A review of chronic granulomatous disease. Adv Ther 2017: 34: 2543-57.

4. Guinea J. Updated EUCAST clinical breakpoints against Aspergillus, implications for the clinical microbiology laboratory. J Fungi (Basel) 2020; 6: 343.

5. Mortensen KL, Knudsen JB, Jensen-Fangel S, Stausbol-Gron B, Arendrup MC, Petersen E. Successful management of invasive aspergillosis presenting as pericarditis in an adult patient with chronic granulomatous disease. Mycoses 2011; 54: e233-6.

6. Camanni G, Sgrelli A, Ferraro L. Aspergillus myofasciitis in a chronic granulomatous disease patient: first case report. Infez Med 2017; 25: 270-3.

7. Elebute M, Massey E, Benjamin S, Stanworth S, Navarrete C, Lucas G. Clinical guidelines for the use of granulocyte transfusions. 2010; cited Feb 2021. www.blood.co.uk/hospitals/library/

8. Valentini CG, Farina F, Pagano L, Teofili L. Granulocyte transfusions: a critical reappraisal. Biol Blood Marrow Transplant 2017; 23: 2034-41.

9. Marciano BE, Allen ES, Conry-Cantilena C, et al. Granulocyte transfusions in patients with chronic granulomatous disease and refractory infections: the NIH experience. J Allergy Clin Immunol 2017; 140: $622-5$.

10. Bielorai B, Toren A, Wolach B, et al. Successful treatment of invasive aspergillosis in chronic granulomatous disease by granulocyte transfusions followed by peripheral blood stem cell transplantation. Bone Marrow Transplant 2000; 26: 1025-8.

11. Sutrave G, Gottlieb DJ. Adoptive cell therapies for posttransplant infections. Curr Opin Oncol 2019; 31: 574-90.

DOI: https://doi.org/10.1016/j.pathol.2021.05.098

\section{Systemic mastocytosis associated with two clonal haematological neoplasms}

Sir,

Systemic mastocytosis with an associated haematological neoplasm (SM-AHN) is recognised by the 2017 World Health Organization (WHO) classification as a subtype of $\mathrm{SM}$ and requires both criteria for SM and AHN to be met as distinct entities. ${ }^{1}$ The second non-mast cell malignancy is generally of myeloid origin with chronic myelomonocytic leukaemia (CMML) being the most common. ${ }^{1,2}$ Other studies have also described the second myeloid neoplasm harbouring the malignant mast cells. There are a few reports of lymphoid neoplasms such as multiple myeloma or lymphoma being associated with SM. ${ }^{1,2}$ In our correspondence, we describe a case of SM associated with two clonal haematological neoplasms, one myeloid and the other a primary plasma cell neoplasm.

A 72-year-old male with severe osteoporosis was found to have an IgM lambda paraprotein of $9 \mathrm{~g} / \mathrm{L}$ on screening tests. A bone marrow biopsy performed showed less than $10 \%$ lambda light chain restricted plasma cells, consistent with a diagnosis of IgM monoclonal gammopathy of undetermined 